\title{
Research on Target Polarization Recognition and Classification Based on BP Neural Network
}

\author{
Bang WU, Qi JIA*, Wei-dong XU, Xu-liang LV and Jiang-hua HU \\ College of Field Engineering, PLA University of Science and Technology, \\ Nanjing, 210007, China \\ *E-mail: jiaqi1983@aliyun.com
}

\begin{abstract}
Polarization detection technology has become an important means of detection technology. This paper discusses the principle and measuring method of polarization detection, typical features and the polarization characteristics, such as rock, concrete, soil, vegetation and military targets. According to the feature of polarization information, the model of target polarization recognition and classification based on BP neural network is constructed. And to validate the precision of this recognition and classification method $t$ the experiment is organized. The results show that the scattering light polarization information as the classification basis is reliable and the model accuracy reached more than $85 \%$, which have a good performance and can be used in many areas.
\end{abstract}

Keywords-Polarization Detection; Stokes Parameter, BP neural network; Recognition.

\section{INTRODUCTION}

Studies show that the polarization image can effectively distinguish between metal and nonmetal, such as artificial target and natural. Polarization detection as a beneficial supplement of intensity detection, can put the amount of information from the $3 \mathrm{~d}$ (light intensity, spectral and spatial) expanded to seven dimensions (light intensity, spectral, spatial, polarization degree, polarization azimuth Angle, polarization ellipticity and rotational direction) [1], helps to improve the accuracy of target detection and feature recognition [2].

This paper mainly through the study of rock, concrete, soil, vegetation and so on typical features and the polarization characteristics of military targets information, the polarization information as the BP neural network input layer information and data for weight calculation, through the activation function and the output, so as to realize classification based on the scattering characteristics of polarization information of target recognition.

\section{PRINCIPLE OF POLARIZATION}

Propagation along the $\mathrm{Z}$ direction of the incident beam, and the $\mathrm{Z}$ direction perpendicular two components (such as $x, y$ axis component) of time average as the beam of light polarization state, which as shown in Figure 1.

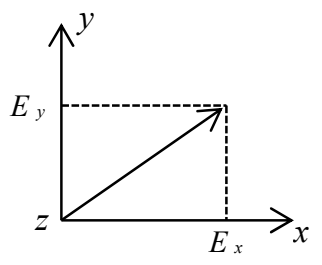

Figure 1 Figure vector method

Polarization of the incident beam characteristics common Jones vector characterization, Stokes vector[3] and Bangka representation. Jones vector can only be said all polarized light, Bangka ball notation is introducing as $s_{0}$ = 1 ball to Stokes space, so this article use Stokes column matrix to represent the polarization of the incident light wave characteristics.

Stokes vector can be expressed with four parameters, , as in Eq. (1).

$$
S=\left[s_{0}, S_{1}, S_{2}, S_{3}\right]^{T}
$$

Where, $s_{0}=<\tilde{E}_{x}^{2}(t)>+<\tilde{E}_{y}^{2}(t)>, s_{1}=<\tilde{E}_{x}^{2}(t)>-<\tilde{E}_{y}^{2}(t)>$, $s_{2}=<2 \tilde{E}_{x}(t) \tilde{E}_{y}(t) \cos \delta>, s_{3}=<2 \tilde{E}_{x}(t) \tilde{E}_{y}(t) \sin \delta>\cdot$

Where, $s_{0}$ said polarized incident light intensity; $s_{1}$ said the $\mathrm{x}$ axis linear polarized light weight; $s_{2}$ said $45^{\circ}$ the direction of the linear polarized light weight; $s_{3}$ said righthanded circularly polarized light weight; For the electric vector in the selected coordinate system along the $x, y$ component; As the phase difference; $<>$ for the time average.

In visible light and near infrared band, non-metallic materials of solar radiation reflected light of circularly polarized light composition is approximate to zero, so, for general features, often will $s_{3}$ parameter is omitted, only need to determine the stokes parameters of $s_{0}$ and $s_{1}, s_{2}$ three parameter can determine the state of polarization.

The principle of polarization imaging detection can be described by Mueller matrix[4]. The role of incident light vector for $\mathbf{S}_{\text {in }}$, Mueller matrix form can be represented as in Eq. (2).

$$
\mathbf{S}_{\text {out }}=\mathbf{M} \cdot \mathbf{S}_{\text {in }}
$$


Where, $\mathbf{M}$ for Mueller matrix, the optical element to evaluate the parameters of Stokes parameter transmission capacity, each optical element can be expressed as a Mueller matrix, each matrix multiplication, we get the Mueller matrix of the whole system, and then through the Stokes parameters inversion calculation to obtain the system output, and then get the target polarization informationas in Eq. (3).

$$
\begin{aligned}
& \psi=\frac{1}{2} \arctan \frac{S_{1}}{S_{2}} \\
& p=\sqrt{S_{1}^{2}+S_{2}^{2}} / S_{0}
\end{aligned}
$$

There are three kinds of Stokes parameter measurement method: Picking method $\left(0^{\circ}, 45^{\circ}, 90^{\circ}\right)$, improve the method of Picking $\left(0^{\circ}, 45^{\circ}, 90^{\circ}, 135^{\circ}\right)$, as well as Fessenkov method $\left(0^{\circ}, 60^{\circ}, 120^{\circ}\right)$.

\section{Polarization Measurement}

Features of polarization reflection measured with multi-angle polarization spectral reflectance measuring instrument, divide into two kinds of indoor and outdoor.Indoor multi-angle polarization spectral reflectance measuring instrument developed by the Chinese academy of sciences Changchun optical precision machinery research institute. By the light source system, second to the (polarization) reflex photometer system and control system of three parts.

Outdoor polarization angles measurement device generally all by this model can achieve multi-angle measurement device with the spectrometer, in front of the spectrometer load polarizing prism to achieve multi-angle polarization measurement [5]. The measurement principle is as shown in Figure 2.In Diagram, $\theta_{\mathrm{i}}$ for the incident zenith Angle, $\theta_{\mathrm{r}}$ for detecting zenith Angle, $\psi_{\mathrm{r}}$ for detecting azimuth Angle, and $0^{\circ}<\theta_{\mathrm{i}}<90^{\circ}, 0^{\circ}<\theta_{\mathrm{r}}<90^{\circ}, 0^{\circ}<\psi_{\mathrm{r}}<360^{\circ}$.

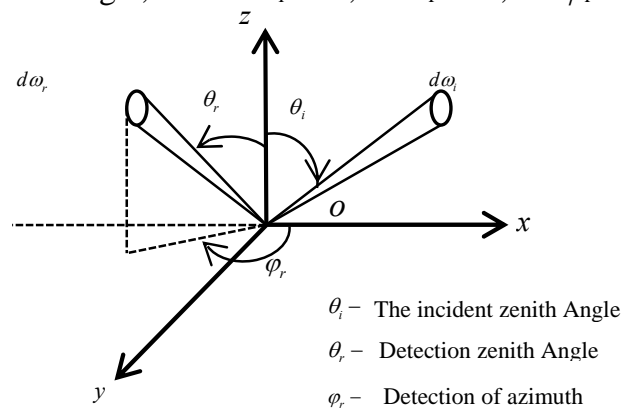

Figure 2 Measuring principle diagram

\section{POLARIZATION PROPERTIES OF TARGETS}

Concrete reflectivity and the polarization of the reflectivity varies with the wavelength increases, but they are not exactly the same change trend. After the observation, the concrete polarized reflectance is lesser, almost to zero, and is not sensitive to observation zenith Angle. Prior to observation, with the increase of the solar zenith Angle, polarized reflectance increases
quickly.When observing zenith Angle certain soil reflectance with wavelength increases, the polarized reflectance basic does not vary with wavelength. When the band phase at the same time, in the observation direction, the polarization degree is small, almost zero, in the front in the direction of observation polarization degree increased with the increase of observing zenith Angle increases.

Most rock experimental data show that the Angle of incidence in less than $20^{\circ}$ small Angle of incidence, there is no mirror, incident Angle is greater than or equal to $30^{\circ}$ large Angle of incidence, rock surface mirror reflection. The reflection spectrum of the rock in the degree of polarization is began with the increase of incident Angle, to the Brewster Angle reaches the maximum, then decreased gradually.Canopy structure and the relationship of plant canopy polarization reflection characteristics is mainly on the relationship between the blade Angle and polarization. Studies have shown that both has the very good ground sex. With the increase of blade Angle, polarization of the plant canopy reflectance also gradually increased.

In detecting Angle, same, light incidence zenith Angle under different conditions, with the increase of incidence zenith Angle, polarized reflectance also increase; Under the condition of light incident zenith Angle is certain, polarization of the plant leaves simple reflection ratio increases with the increase of detection zenith Angle, the maximum in the azimuth Angle of $180^{\circ}$.To observation in the polarization of the reflectivity is small, after close to $15^{\circ}$ polarized reflectance minimum, while the former to the polarized reflectance is bigger.

\section{TARGET POLARIZATION INFORMATION IDENTIFICATION}

The BP neural network is a kind of artificial neural network based on error feedback [6, 7], by an input layer, one or more of the hidden layer and one output layer.BP neural network is characterized by: a layer of neurons can't cross connect another layer, a layer of neurons, the error feedback can only step by step.The handling process of BP neural network is mainly information or data input through the input layer in, through computing the activation function is passed on to the hidden layer, and then calculate the hidden layer nodes information is passed on to the output node, it is to get the final output. BP neural network to accord with the result of expert experience only output, to does not conform to the requirements of data and information back to continue to process, so in the BP network, the transmission of information is positive, the error propagation is backward, so also called back propagation algorithm $[8,9]$. 


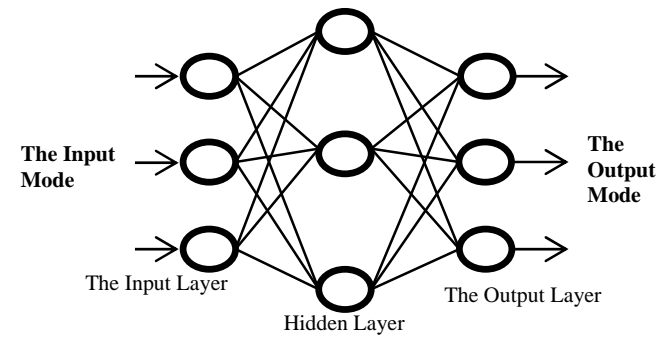

Figure 3 Three layers BP network

The polarization spectral information has better classification ability. Remote sensing image classification accuracy of the evaluation are classified by the figure compared with the actual data, with the percentage of correct classification accuracy.Currently widely used method is to use chaos matrix expression classification accuracy, chaotic matrix shows the real feature data sample classification results and cross distribution [10]. In chaotic matrix each column represents the reference data for each kind of sample number, each row number of each type of sample classification data, main diagonal expressed the correct classification of the pixel.Overall, the BP neural network classification method is compared with other methods of supervised classification and unsupervised classification method, has certain advantages, not only to the sample of strong adaptability, but also has good reliability, has certain requirements, but the number of samples in the sample size, high precision, but time is long, the small sample size, could not be very good to the learning and adjustment of parameters, precision is affected, even failure classification as a result of learning problem.

\section{EXPERIMENT}

In order to validate the correctness of the model, we organized the experiment. 120 target are selected to calculated and experimented. The experiment is done in eastern suburb of Nanjing city. After a large number training data, the precision of the classification and identify the targets is validated. The experiment results show that the model accuracy reached more than 85\%, which have a good performance and can be used in many application areas.

\section{CONCLUSION}

This paper discusses the principle and measuring method of polarization detection, summarizes rock, concrete, soil, vegetation and other typical features and ships and other military targets polarization information, and according to the characteristics of different ground objects on the surface scattering light polarization analysis results, based on the BP neural network, through input the polarization information of a given material, implements the qualitative identification and classification, based on the material of scattering light polarization identification provides experimental support. The results show that the scattering light polarization information as the classification basis is reliableandthe model accuracy has a good performance.

\section{ACKNOWLEDGMENT}

This work is supported by projects grant from National Key Laboratory on Electromagnetic Environment Effects and Electro-optical Engineering (Grand NO. FD2013009 and FD2015011).

Work partially supported by youth fund of College of Field Engineering, PLA University of Science and Technology.

\section{REFERENCES}

[1] D. Clarke,J. F. Grainger, Polarrized Light and Optical Measurement, (Oxford, Pergamon Press,1971).

[2] X. B.Sun, Y. L Qiao, H. Jin, Artificial target polarization characteristics experimental study,High technology communication, 13 (2013).

[3] D. I. Huige, H. P. Zeng, Z. P. He, The High-precision Measurement of the Polarization of Light Based on Division-ofamplitude,Opto-Electronic Engineering, 39(2012).

[4] Y. B. Li, S. B. Li, W. J. Chen, A Microscope Measurement System of Cellular Mueller Matrix,Opto-Electronic Engineering,37(2010).

[5] W. T. Yei, Polarization remote sensing physical,(Beijing, Science Press, 2014).

[6] H. Jian, G. P.Wu, BP neural network based on K - L transform remote sensing image classification, Journal of surveying and mapping science, 3 (2009).

[7] Y. S. Zhang, X. Z. Feng, R. Z. Ruan, BP neural network based on GIS remote sensing image classification study, Journal of Nanjing university, 33 (2003).

[8] Y. H. Jia, Artificial neural network in the application of multisource remote sensing image classification,Bulletin of surveying and mapping, 7 (2000).

[9] W. Chong, W. B. Wu, J. Ping, Remote sensing image classification method based on BP neural network,Journal of Liaoning engineering technology university, 28 (2009).

[10] Y. Q. Zhao, P.Quan, Y. M.Cheng,Polarization imaging spectral remote sensing and application, (Beijing, National Defense Industry Press, 2011). 